\title{
Multilevel Analysis of Air Pollution and Early Childhood Neurobehavioral Development
}

\author{
Ching-Chun Lin ${ }^{1}$, Shih-Kuan Yang ${ }^{1}$, Kuan-Chia Lin ${ }^{2}$, Wen-Chao Ho ${ }^{3}$, Wu-Shiun Hsieh ${ }^{4}$, \\ Bih-Ching Shu ${ }^{5}$ and Pau-Chung Chen ${ }^{1,6,7, *}$
}

1 Institute of Occupational Medicine and Industrial Hygiene, National Taiwan University College of Public Health, 17 Syujhou Road, Taipei 10055, Taiwan; E-Mails: ccleuven@gmail.com (C.-C.L.); skyang530@gmail.com (S.-K.Y.)

2 Department of Nursing, National Taipei University of Nursing and Health Sciences,

Taipei 10845, Taiwan; E-Mail: kuanchia@ntunhs.edu.tw

3 Institute of Environmental Medicine, China Medical University, Taichung 40402, Taiwan;

E-Mail:wcho@mail.cmu.edu.tw

4 Department of Pediatrics, National Taiwan University College of Medicine and Hospital, Taipei 10055, Taiwan; E-Mail: hsiehws@ntu.edu.tw

5 Institute of Allied Health Sciences and School of Nursing, National Cheng Kung University, Tainan 701, Taiwan; E-Mail: shubih@mail.ncku.edu.tw

6 Department of Public Health, National Taiwan University College of Public Health, Taipei 10055, Taiwan

7 Department of Environmental and Occupational Medicine, National Taiwan University College of Medicine and Hospital, Taipei 10055, Taiwan

* Author to whom correspondence should be addressed; E-Mail: pchen@ntu.edu.tw; Tel.: +886-2-3366-8088; Fax: +886-2-2358-2402.

Received: 15 August 2013; in revised form: 19 June 2014 / Accepted: 23 June 2014 / Published: 2 July 2014

\begin{abstract}
To investigate the association between the ambient air pollution levels during the prenatal and postnatal stages and early childhood neurobehavioral development, our study recruited 533 mother-infant pairs from 11 towns in Taiwan. All study subjects were asked to complete childhood neurobehavioral development scales and questionnaires at 6 and 18 months. Air pollution, including particulate matter $\leq 10 \mu \mathrm{m}\left(\mathrm{PM}_{10}\right)$, carbon monoxide (CO), sulfur dioxide $\left(\mathrm{SO}_{2}\right)$, nitrogen dioxide $\left(\mathrm{NO}_{2}\right)$, ozone $\left(\mathrm{O}_{3}\right)$, and hydrocarbons, was measured at air quality monitoring stations in the towns where the subjects lived. Multilevel
\end{abstract}


analyses were applied to assess the association between air pollution and childhood neurobehavioral development during pregnancy and when the children were 0 to 6 months, 7 to 12 months, and 13 to 18 months old. At 18 months, poor subclinical neurodevelopment in early childhood is associated with the average $\mathrm{SO}_{2}$ exposure of prenatal, during all trimesters of pregnancy and at postnatal ages up to 12 months (first trimester $\beta=-0.083$, $\mathrm{se}=0.030$; second and third trimester $\beta=-0.114$, se $=0.045$; from birth to 12 months of age $\beta=-0.091$, se $=0.034$ ). Furthermore, adverse gross motor below average scores at six months of age were associated with increased average non-methane hydrocarbon, (NMHC) levels during the second and third trimesters $(\beta=-8.742$, se $=3.512)$. Low-level $\mathrm{SO}_{2}$ exposure prenatally and up to twelve months postnatal could cause adverse neurobehavioral effects at 18 months of age. Maternal NMHC exposure during the 2 nd and 3rd trimesters of pregnancy would be also associated with poor gross motor development in their children at 6 months of age.

Keywords: air pollution; neurobehavioral development; trimester; multilevel analysis

\section{Introduction}

Previous studies found that outdoor air pollution has neurotoxic and behavioral effects on humans or animals. Carbon monoxide (CO) interferes with oxygen transport through the formation of carboxyhemoglobin and exposure to carboxyhemoglobin levels of $2.1 \%$ to $4.2 \%$. Carboxyhemoglobin resulted in a significant decrease in video game performance and may impair humans' higher cognitive functions [1]. Even low-level exposure to $\mathrm{CO}$ impairs higher cognitive functions [2]. In animal studies, prenatal exposure to nitrogen dioxide $\left(\mathrm{NO}_{2}\right)$ affected neuromuscular coordination and caused functional deficits in the developing offspring of mice [3] and prenatal sulfur dioxide $\left(\mathrm{SO}_{2}\right)$ exposure reduced the levels of several mouse social/agonistic behaviors, such as tail rattling, freezing, and defensive postures [4] impaired neuromuscular coordination [5] and impaired the coordination and locomotor development of their offspring [6]. Exposure to high ozone $\left(\mathrm{O}_{3}\right)$ concentrations during gestation induces permanent cerebellar damage in rats and negatively impacts activity/exploration and social behavior [7,8]. It is also found the significant increase in freezing and defensive postures, decreases in nose-sniffing behavior and decreased nerve growth factor (NGF) levels in the hippocampus [9].

In humans evidence shows that some of the pollutants can affect the developing brain and adversely affect cognitive development [10] PAH pollutants can exert genotoxic effects which inducing mutations as epigenetic effects [11]. Molecular epidemiologic studies also found that the fetus is more susceptible than adults to the effects of PAH and other toxicants [12].

The PAH-DNA adducts in cord blood were associated with delay of developmental $[10,13,14]$, IQ [11], child behavior symptom scores of Anxious/Depressed, and Attention Problems [15]. In addition, higher levels of cord PAH-DNA adducts have been associated with reduced scores on neurocognitive tests, alone or in combination with environmental tobacco smoke (ETS) [10,11,15-17]. 
There is limited evidence on the relationship between air pollutant exposure and early childhood neurodevelopment, and the neurological effects of air pollution remain unclear [18]. The developing nervous system is an extremely sensitive target, and the blood-brain barrier is not fully developed until the middle of the first year of life [19]. Our study aimed to investigate the relationship between exposure to ambient air pollutants during the prenatal and postnatal periods and with early childhood neurodevelopment.

\section{Material and Methods}

\subsection{Study Population}

A prospective cohort study was conducted between October 2003 and January 2004 as pilot survey of Taiwan Birth Cohort Pilot Study (TBCS-p). The participants of TBCS-p were randomly selected from babies born between October 2003 and January 2004 in 29 villages or cities in Taiwan and were followed up when they were 6,18 months old. There are 629 mother-infant pairs agreed to participate in the study. There were 613 mother-infant pairs from 11 towns with air quality monitoring stations operated by the Taiwan Air Quality Monitoring Network (TAQMN) [20]. We excluded infants whose mothers smoked during the gestational period $(N=10)$ and infants with congenital defects $(N=22)$, preterm birth $(N=31)$, or low birth weight $(N=33)$. A total of 533 mother-infant pairs were recruited for this study. All study participants provided prior informed consent, as required by the Ethics Review Board of the National Taiwan University College of Public Health.

\subsection{Exposure Assessment}

The TAQMN was established in 1993 by the Environmental Protection Administration. Ambient levels of key air pollutants are measured and reported on an hourly basis. All instruments in TAQMN stations are maintained and periodically calibrated according to the TAQMN quality management plan. TAQMN monitoring stations provide hourly ambient concentrations of particulate matter $\leq 10 \mu \mathrm{m}$ $\left(\mathrm{PM}_{10}\right), \mathrm{CO}, \mathrm{O}_{3}, \mathrm{SO}_{2}, \mathrm{NO}_{2}$, and total hydrocarbons (THCs) and nonmethane hydrocarbons (NMHCs). In our study, hourly data were collected from the 11 air-quality monitoring stations in the towns where the study subjects inhabited.

At those monitoring stations, $\mathrm{CO}$ was measured by nondispersive infrared photometry (RFCA-0981-054, Thermo Environmental Instruments, Franklin, MA, USA); $\mathrm{O}_{3}$ was measured by ultraviolet photometric analysis (TNMH 642, Thermo Environmental Instruments); $\mathrm{PM}_{10}$ was measured by beta-ray absorption; $\mathrm{SO}_{2}$ was measured by ultraviolet fluorescence; $\mathrm{NO}_{2}$ was measured using a chemiluminescence analyzer (Thermo Environmental Instruments Model 42C or ECOTECH-ML 9841A, detection limit $0.4 \mathrm{ppb}$ ); and THCs and NMHCs were measured using a flame-ionization detector (FID; DANI Instruments-TNMH 462, Franklin, MA, USA).

The air pollutant exposure data for each child were linked from the air-quality monitoring stations of town to the exposure period from the beginning of the gestational period to eighteen months after birth. To observe the relationship between timing and magnitude of air pollutant exposure, the gestational period was divided into three trimesters of three calendar months from the mothers' last 
menstrual period (LMP) to their children's birthdays, and the period after delivery was also divided into three stages: birth to 6 months, 7 to 12 months, and 13 to 18 months for each study subject.

The traffic peaks are at around the 7 a.m and the 7 p.m. Normally, baby children would stay at home or the nearby day care center, and the outdoor activity of the children are during the daytime. We considered the daytime ( 7 a.m. to 7 p.m.) average levels of $\mathrm{CO}, \mathrm{O}_{3}, \mathrm{PM}_{10}, \mathrm{SO}_{2}, \mathrm{NO}_{2}, \mathrm{THC}$, and NMHC among different stages as our subjects' exposure levels. If more than $25 \%$ of the daily average air pollutant data was missing among different stages, we did not calculate average air pollutant levels because these data might not reliably represent exposure levels during that period.

\subsection{Data Collection}

Subjects' demographic data were obtained from the Taiwan national birth registry and the interview questionnaire. Birth certificate forms contain the following information: mother's residential county and town, date of registration, parental identification number, birthday, education level, and marital status, and the infant's neonatal identification number, date of birth, birth weight, gestational age, parity, singleton or multiple birth, place of delivery (hospital or clinic), and the midwife's or obstetrician's information. We used the infants' dates of birth and gestational ages recorded on the birth certificates to estimate the mothers' last menstrual period (LMP) and their children's exposure periods. Information about maternal age, maternal education level, paternal education level, nursery type (which by parents, grandparents or others), and exposure to environmental tobacco smoke were also collected by trained interviewers using structured questionnaires, and response by the parents or the main care person at the children's age of 6 and 18 months separately.

\subsection{Neurobehavioral Development}

The Birth Cohort Study (TBCS) was to develop an efficient developmental screening instrument for interviews in clinical and community settings. The purpose of a developmental screening tool is to survey the development of a child. It is a preventive approach to detect the problem before the child becomes deviant, so that every child can develop to his or her fullest potential the TBCS scale can be easily completed by the majority of parents. The TBCS can be used as the first stage of a two-stage developmental screening process.

The TBCS scale is a parent-reported measure of a child's neurodevelopmental performance that consists of four developmental dimensions: gross motor, fine motor, language/communication (language), and social/self-care abilities (social). Parents were given two neurobehavioral development scales to complete at each interview. The scales contain 38 and 17 items each. Response choices consist of three ordinal categories relating to frequency (never, sometimes, and all the time). A response of "never" received one point, and "all the time" received three points. We added the scores in each dimension for each child. Both the 6- and 18-month scales have good predictive validity, and these dimensions correlate with the second edition of the Bayley Scales of Infant Development (BSID-II) [21,22]. 


\subsection{Statistical Analysis}

The hierarchical linear models (HLM) can be used to analyze the relationships between community level environmental data, individual risk factors, and birth outcomes. HLM is often used to study the relationship between community level factors and individual outcomes in health outcomes. Because our data were hierarchically organized and all air pollutant data were nested within different geographic areas, the associations between air pollutant exposure levels and childhood neurodevelopment were assessed via multilevel modeling analysis [23].

The analyses included individual (level one) and environmental conditions of area levels (level two). The individual level were personal variable include maternal education, maternal nationality, environmental tobacco smoke exposure, infant gestational age, breastfeeding, and parental nursery type. The area level was area air pollutant data. Using the combined level-1 and level-2 data sets, HLM provides a way to analyze the degree to which the effects of individual level variables in birth data vary systematically or randomly as a function of level-2 variables [24].

We evaluated the association between air pollutant levels during each period and neurodevelopment at 6 and 18 months of age. All analyses included individual and town exposure levels. The air pollutant and neurodevelopment data were continuous variables. The covariates for each model included maternal education (college or beyond, senior high, junior high or elementary), maternal nationality (Taiwanese, non-Taiwanese), environmental tobacco smoke exposure (never, ever), infant gestational age (weeks), breastfeeding (less than 6 months, 6 months or more), and parental nursery type. These statistical analyses were performed using SPSS for Windows, Version 11.0 (SPSS Inc., Chicago, IL, USA).

\section{Results}

The characteristics for 533 singleton live births are presented in Table 1. Most mothers in this study had high education levels, including 242 who stated they were college graduates (45.4\%) and 219 senior high school graduates (41.1\%), and $14.1 \%$ of mothers recruited for this study were foreign. The mean gestational age was 38.8 weeks, the mean birth weight was $3190.7 \mathrm{~g}$, and about half (56.5\%) of the infants were male. Among the study infants, $49.3 \%$ had prenatal environmental tobacco exposure and only $17.4 \%$ were breastfed for more than six months.

We found that gross motor scores at 6 months of age were negatively associated with the NMHC levels during the second and third trimesters. In addition, fine motor scores at 18 months of age were negatively associated with the average $\mathrm{SO}_{2}$ exposure levels during pregnancy and in children from birth to 12 months of age.

Table 2 shows the average air pollutant level profiles for each period. Except for ozone, all other air pollutants had an obvious peak average level between the infants' births and six months of age. Conversely, air pollutants levels were low during the second trimester and the six to twelve month period. Most pollutants exhibit clear seasonal variations, with higher concentrations in the fall and winter.

The multilevel analysis of the relationship between air pollutant exposure and neurobehavioral development scores for children at six months and eighteen months of age are shown in Tables 3 and 4. At six months, the increased NMHC exposure during the second and third trimesters in utero 
was associated with adverse gross motor scores $(\beta=-8.742$, se $=3.512)$. No other pollutants had a significant effect on neurobehavioral development performance at six months.

Table 1. Characteristic of the study subjects.

\begin{tabular}{lc}
\hline \multicolumn{1}{c}{ Characteristics } & Study Subjects \\
\hline Total & 533 \\
Maternal education, N (\%) & \\
$\quad$ College + & $242(45.4)$ \\
$\quad$ Senior high & $219(41.1)$ \\
$\quad$ Junior high & $52(9.8)$ \\
Elementary & $19(3.6)$ \\
Maternal nationality, N (\%) & \\
$\quad$ Taiwanese & $458(85.9)$ \\
Foreign & $75(14.1)$ \\
Gestational age, mean (SD) & $38.8(1.2)$ \\
Breast feeding ${ }^{\text {a }}, \mathrm{N}(\%)$ & \\
$\quad \geq 6$ months & $93(17.4)$ \\
0-5 months & $440(82.6)$ \\
Environmental Tobacco Smoke (\%) & \\
$\quad$ Yes & $263(49.3)$ \\
$\quad$ No & $270(50.7)$ \\
Gender, N (\%) & \\
$\quad$ Male & $301(56.5)$ \\
Female & $232(43.5)$ \\
Birth weight, mean (SD) & \\
Parity, N (\%) & \\
1 & \\
2 &
\end{tabular}

${ }^{a}$ breast feeding was defined as including mixed and exclusive breast feeding.

At eighteen months, the increased $\mathrm{SO}_{2}$ exposure among the three trimesters of pregnancy and the period between birth and twelve months of age were significantly associated with a decrease in fine motor development performance scores at 18 months (first trimester $\beta=-0.083$, se $=0.030$; second and third trimester $\beta=-0.114$, se $=0.045$; birth to twelve months $\beta=-0.091$, se $=0.034$ ). $\mathrm{SO}_{2}$ exposure from thirteen months to eighteen months after birth was not significantly associated with fine motor scores. Aside from $\mathrm{SO}_{2}$, all other air pollutants in this study were not significantly associated with neurobehavioral developmental scores at 18 months of age. The $\mathrm{SO}_{2}$ exposure during all trimesters of pregnancy and the effect from birth to 12 months on fine motor development scores at eighteen months. The prediction line shows an adverse association between increasing $\mathrm{SO}_{2}$ exposure levels and fine motor scores. 
Table 2. Mean and standard deviations of air pollutant concentrations at different periods.

\begin{tabular}{lcrr|rrr|rrr}
\hline \multirow{2}{*}{ Air Pollutants } & \multicolumn{3}{c}{ First Trimester } & \multicolumn{3}{c|}{ Second Trimester } & \multicolumn{3}{c}{ Third Trimester } \\
\cline { 2 - 10 } & \multicolumn{1}{c}{ Range } & Mean & \multicolumn{1}{c|}{ SD } & \multicolumn{1}{c|}{ Range } & Mean & SD & Range & Mean & SD \\
\hline $\mathrm{CO}(\mathrm{ppm})$ & $0.33-0.93$ & 0.63 & 0.14 & $0.24-0.71$ & 0.48 & 0.11 & $0.36-0.90$ & 0.58 & 0.13 \\
$\mathrm{O}_{3}(\mathrm{ppb})$ & $29.86-52.52$ & 38.87 & 5.30 & $19.07-49.18$ & 34.32 & 6.35 & $22.65-62.84$ & 37.45 & 10.18 \\
$\mathrm{PM}_{10}\left(\mathrm{~g} / \mathrm{m}^{3}\right)$ & $31.22-36.88$ & 59.26 & 13.51 & $23.61-54.47$ & 39.41 & 6.63 & $31.21-107.52$ & 55.88 & 19.58 \\
$\mathrm{SO}_{2}(\mathrm{ppb})$ & $0.27-14.63$ & 4.45 & 2.50 & $0.28-6.86$ & 3.63 & 1.36 & $0.25-13.31$ & 4.08 & 2.51 \\
$\mathrm{NO}_{2}(\mathrm{ppb})$ & $6.41-28.33$ & 18.17 & 5.62 & $6.16-23.60$ & 12.57 & 4.18 & $7.70-32.25$ & 18.33 & 4.93 \\
$\mathrm{THC}(\mathrm{ppm})$ & $2.22-3.05$ & 2.52 & 0.22 & $2.03-2.78$ & 2.37 & 0.18 & $2.31-3.19$ & 2.59 & 0.17 \\
$\mathrm{NMHC}(\mathrm{ppm})$ & $0.19-0.58$ & 0.38 & 0.10 & $0.15-0.68$ & 0.33 & 0.14 & $0.16-0.98$ & 0.35 & 0.14 \\
\hline
\end{tabular}

\begin{tabular}{lcrc|ccc|ccc}
\hline \multirow{2}{*}{ Air Pollutants } & \multicolumn{3}{c|}{ 1-6 Months } & \multicolumn{3}{c|}{ 7-12 Months } & \multicolumn{3}{c}{ 13-18 Months } \\
\cline { 2 - 10 } & \multicolumn{1}{c}{ Range } & Mean & \multicolumn{1}{c|}{ SD } & Range & Mean & SD & Range & Mean & SD \\
\hline $\mathrm{CO}(\mathrm{ppm})$ & $0.37-0.93$ & 0.66 & 0.14 & $0.21-0.71$ & 0.45 & 0.11 & $0.40-1.01$ & 0.67 & 0.14 \\
$\mathrm{O}_{3}(\mathrm{ppb})$ & $27.95-48.91$ & 36.64 & 5.46 & $22.38-49.06$ & 35.85 & 6.90 & $26.34-41.25$ & 31.51 & 3.74 \\
$\mathrm{PM}_{10}\left(\mu \mathrm{g} / \mathrm{m}^{3}\right)$ & $36.37-116.58$ & 72.39 & 19.80 & $38.96-71.52$ & 54.95 & 8.68 & $37.66-111.78$ & 69.79 & 17.45 \\
$\mathrm{SO}_{2}(\mathrm{ppb})$ & $0.47-12.72$ & 5.21 & 2.67 & $1.09-9.26$ & 4.66 & 2.01 & $2.54-15.26$ & 6.31 & 3.18 \\
$\mathrm{NO}_{2}(\mathrm{ppb})$ & $9.15-32.13$ & 23.10 & 6.25 & $6.76-24.56$ & 15.98 & 5.44 & $9.29-32.00$ & 21.12 & 6.47 \\
$\mathrm{THC}(\mathrm{ppm})$ & $2.46-2.92$ & 2.70 & 0.15 & $1.99-2.85$ & 2.32 & 0.30 & $1.97-2.56$ & 2.20 & 0.20 \\
$\mathrm{NMHC}(\mathrm{ppm})$ & $0.20-0.63$ & 0.42 & 0.13 & $0.13-0.57$ & 0.31 & 0.17 & $0.15-0.49$ & 0.28 & 0.11 \\
\hline
\end{tabular}

The average wind speed during the study period (from 2003 to 2005) was $2.00 \mathrm{~m} / \mathrm{s}$ (range: $4.36 \mathrm{~m} / \mathrm{s}$ to $0.68 \mathrm{~m} / \mathrm{s}$ ). The average ambient temperature during the study period was $23.77{ }^{\circ} \mathrm{C}$ (range: $31.44{ }^{\circ} \mathrm{C}$ to $12.51{ }^{\circ} \mathrm{C}$ ). The average relative humidity during the study period was $72.13 \%$ (range: $84.38 \%$ to $32.03 \%$ ). 
Table 3. Multilevel analysis of single air pollutants and neurodevelopment scores at six months of age.

\begin{tabular}{|c|c|c|c|c|c|c|c|c|c|c|c|}
\hline \multirow{2}{*}{\multicolumn{2}{|c|}{ Air Pollutant in the Different Periods }} & \multicolumn{2}{|c|}{ Gross Motor } & \multicolumn{2}{|c|}{ Fine Motor } & \multicolumn{2}{|c|}{ Language } & \multicolumn{2}{|c|}{ Social-Personal } & \multicolumn{2}{|c|}{ Total } \\
\hline & & $\beta$ & SE & $\beta$ & SE & $\boldsymbol{\beta}$ & SE & $\boldsymbol{\beta}$ & SE & $\beta$ & SE \\
\hline \multirow[t]{3}{*}{$\mathrm{CO}$} & 1st trimester & 1.414 & 1.350 & -0.088 & 0.736 & -0.166 & 0.860 & 0.338 & 0.932 & 1.655 & 2.764 \\
\hline & 2nd \& 3rd trimester & 0.459 & 1.695 & -0.243 & 0.928 & -0.655 & 1.088 & -0.180 & 1.223 & -0.528 & 3.477 \\
\hline & Birth-6 months & 0.100 & 1.324 & -0.219 & 0.718 & -0.456 & 0.834 & 0.411 & 0.913 & -0.043 & 2.697 \\
\hline \multirow[t]{3}{*}{$\mathrm{O}_{3}$} & 1st trimester & -0.056 & 0.044 & -0.037 & 0.024 & -0.019 & 0.030 & 0.016 & 0.025 & -0.116 & 0.091 \\
\hline & 2nd \& 3rd trimester & 0.001 & 0.028 & 0.003 & 0.016 & 0.012 & 0.020 & 0.009 & 0.022 & 0.030 & 0.058 \\
\hline & Birth-6 months & 0.078 & 0.052 & -0.042 & 0.026 & -0.011 & 0.032 & 0.007 & 0.036 & 0.058 & 0.082 \\
\hline \multirow[t]{3}{*}{$\mathrm{PM}_{10}$} & 1st trimester & 0.008 & 0.020 & -0.007 & 0.011 & 0.003 & 0.011 & 0.017 & 0.016 & 0.033 & 0.044 \\
\hline & 2nd \& 3rd trimester & 0.005 & 0.024 & -0.023 & 0.015 & -0.006 & 0.018 & -0.007 & 0.021 & -0.022 & 0.050 \\
\hline & Birth-6 months & -0.004 & 0.011 & -0.006 & 0.007 & 0.003 & 0.008 & 0.001 & 0.009 & 0.006 & 0.022 \\
\hline \multirow[t]{3}{*}{$\mathrm{SO}_{2}$} & 1st trimester & 0.015 & 0.072 & -0.002 & 0.042 & 0.027 & 0.050 & 0.010 & 0.056 & 0.090 & 0.147 \\
\hline & 2nd \& 3rd trimester & -0.125 & 0.113 & -0.037 & 0.062 & 0.036 & 0.073 & -0.009 & 0.082 & -0.092 & 0.229 \\
\hline & Birth-6 months & -0.053 & 0.070 & -0.001 & 0.039 & 0.033 & 0.046 & 0.002 & 0.051 & 0.019 & 0.141 \\
\hline \multirow[t]{3}{*}{$\mathrm{NO}_{2}$} & 1st trimester & -0.005 & 0.034 & -0.004 & 0.019 & 0.017 & 0.022 & 0.022 & 0.025 & 0.023 & 0.070 \\
\hline & 2nd \& 3rd trimester & -0.067 & 0.046 & -0.028 & 0.025 & -0.011 & 0.029 & 0.002 & 0.033 & -0.110 & 0.093 \\
\hline & Birth-6 months & -0.002 & 0.031 & -0.010 & 0.016 & 0.002 & 0.019 & 0.012 & 0.021 & 0.002 & 0.062 \\
\hline \multirow[t]{3}{*}{ THC } & 1st trimester & 0.123 & 1.233 & -0.504 & 0.656 & 0.583 & 0.782 & 0.192 & 0.871 & 0.438 & 2.624 \\
\hline & 2nd $\& 3$ rd trimester & -2.143 & 1.888 & 0.940 & 0.979 & 0.269 & 1.203 & -0.970 & 1.335 & -1.606 & 4.031 \\
\hline & Birth -6 months & -0.173 & 1.908 & -1.451 & 0.996 & 0.523 & 1.210 & 0.087 & 1.346 & -0.753 & 4.058 \\
\hline \multirow[t]{3}{*}{ NMHC } & 1 st trimester & -6.134 & 4.392 & -2.014 & 2.471 & 3.121 & 2.957 & -0.673 & 3.284 & -5.721 & 9.843 \\
\hline & 2nd \& 3rd trimester & $-8.742 *$ & 3.512 & -8.742 & 3.512 & 2.091 & 1.985 & 2.091 & 1.985 & -3.986 & 8.024 \\
\hline & Birth-6 months & -2.822 & 2.696 & -2.166 & 1.494 & 1.453 & 1.809 & -0.337 & 2.008 & -4.603 & 5.952 \\
\hline
\end{tabular}

Adjusted for maternal education level, maternal nationality, gestational age, infant sex, breastfeeding, environmental tobacco smoke exposure, and nursery type (which include parents, grandparents or others). Subject: area;* Significant difference $(p<0.05)$. 
Table 4. Multilevel analysis of single air pollutants and neurodevelopment scores at eighteen months of age.

\begin{tabular}{|c|c|c|c|c|c|c|c|c|c|c|c|}
\hline \multirow{2}{*}{\multicolumn{2}{|c|}{ Air Pollutant in the Different Periods }} & \multicolumn{2}{|c|}{ Gross Motor } & \multicolumn{2}{|c|}{ Fine Motor } & \multicolumn{2}{|c|}{ Language } & \multicolumn{2}{|c|}{ Social-Personal } & \multicolumn{2}{|c|}{ Total } \\
\hline & & $\beta$ & SE & $\boldsymbol{\beta}$ & SE & $\boldsymbol{\beta}$ & SE & $\beta$ & SE & $\boldsymbol{\beta}$ & SE \\
\hline $\mathrm{CO}$ & $\begin{array}{l}\text { 1st trimester } \\
\text { 2nd \& 3rd trimester } \\
\text { Birth-12 months } \\
\text { 13-18 months }\end{array}$ & $\begin{array}{r}0.265 \\
-0.059 \\
0.297 \\
0.077\end{array}$ & $\begin{array}{l}0.480 \\
0.603 \\
0.542 \\
0.552\end{array}$ & $\begin{array}{r}0.005 \\
-1.095 \\
-0.236 \\
-0.114\end{array}$ & $\begin{array}{l}0.585 \\
0.726 \\
0.662 \\
0.652\end{array}$ & $\begin{array}{l}1.129 \\
0.177 \\
0.806 \\
0.285\end{array}$ & $\begin{array}{l}0.758 \\
0.947 \\
0.859 \\
0.826\end{array}$ & $\begin{array}{r}0.226 \\
-0.869 \\
-0.053 \\
-1.394\end{array}$ & $\begin{array}{l}0.644 \\
0.799 \\
0.729 \\
0.626\end{array}$ & $\begin{array}{r}1.711 \\
-2.119 \\
0.856 \\
-0.650\end{array}$ & $\begin{array}{l}1.705 \\
2.084 \\
1.928 \\
1.832 \\
\end{array}$ \\
\hline $\mathrm{O}_{3}$ & $\begin{array}{l}\text { 1st trimester } \\
\text { 2nd \& 3rd trimester } \\
\text { Birth-12 months } \\
\text { 13-18 months }\end{array}$ & $\begin{array}{l}-0.012 \\
-0.001 \\
-0.013 \\
-0.014\end{array}$ & $\begin{array}{l}0.017 \\
0.011 \\
0.023 \\
0.024\end{array}$ & $\begin{array}{r}-0.022 \\
-0.015 \\
0.014 \\
0.011\end{array}$ & $\begin{array}{l}0.019 \\
0.013 \\
0.025 \\
0.028\end{array}$ & $\begin{array}{r}-0.018 \\
0.018 \\
0.044 \\
0.017\end{array}$ & $\begin{array}{l}0.024 \\
0.017 \\
0.031 \\
0.035\end{array}$ & $\begin{array}{l}0.030 \\
0.022 \\
0.046 \\
0.033\end{array}$ & $\begin{array}{l}0.017 \\
0.015 \\
0.032 \\
0.033\end{array}$ & $\begin{array}{r}-0.026 \\
0.029 \\
0.112 \\
0.053\end{array}$ & $\begin{array}{l}0.052 \\
0.036 \\
0.084 \\
0.075\end{array}$ \\
\hline $\mathrm{PM}_{10}$ & $\begin{array}{l}\text { 1st trimester } \\
\text { 2nd \& 3rd trimester } \\
\text { Birth-12 months } \\
\text { 13-18 months }\end{array}$ & $\begin{array}{l}-0.004 \\
-0.009 \\
-0.005 \\
-0.004 \\
\end{array}$ & $\begin{array}{l}0.008 \\
0.010 \\
0.006 \\
0.005\end{array}$ & $\begin{array}{l}-0.007 \\
-0.015 \\
-0.011 \\
-0.010 \\
\end{array}$ & $\begin{array}{l}0.008 \\
0.012 \\
0.007 \\
0.005 \\
\end{array}$ & $\begin{array}{l}0.005 \\
0.004 \\
0.004 \\
0.002 \\
\end{array}$ & $\begin{array}{l}0.011 \\
0.012 \\
0.009 \\
0.006 \\
\end{array}$ & $\begin{array}{l}0.018 \\
0.012 \\
0.006 \\
0.003 \\
\end{array}$ & $\begin{array}{l}0.010 \\
0.014 \\
0.009 \\
0.006 \\
\end{array}$ & $\begin{array}{r}0.010 \\
0.005 \\
0.001 \\
-0.003 \\
\end{array}$ & $\begin{array}{l}0.026 \\
0.029 \\
0.020 \\
0.015 \\
\end{array}$ \\
\hline $\mathrm{SO}_{2}$ & $\begin{array}{l}\text { 1st trimester } \\
\text { 2nd \& 3rd trimester } \\
\text { Birth-12 months } \\
\text { 13-18 months }\end{array}$ & $\begin{array}{l}-0.011 \\
-0.040 \\
-0.017 \\
-0.009\end{array}$ & $\begin{array}{l}0.028 \\
0.040 \\
0.031 \\
0.024\end{array}$ & $\begin{array}{l}-0.083 * \\
-0.114 * \\
-0.091 * \\
-0.057\end{array}$ & $\begin{array}{l}0.030 \\
0.045 \\
0.034 \\
0.029 \\
\end{array}$ & $\begin{array}{r}0.019 \\
-0.044 \\
-0.010 \\
-0.021\end{array}$ & $\begin{array}{l}0.043 \\
0.062 \\
0.047 \\
0.034\end{array}$ & $\begin{array}{l}0.036 \\
0.029 \\
0.009 \\
0.017 \\
\end{array}$ & $\begin{array}{l}0.037 \\
0.054 \\
0.041 \\
0.031 \\
\end{array}$ & $\begin{array}{l}-0.026 \\
-0.140 \\
-0.102 \\
-0.073\end{array}$ & $\begin{array}{l}0.093 \\
0.137 \\
0.101 \\
0.075 \\
\end{array}$ \\
\hline $\mathrm{NO}_{2}$ & $\begin{array}{l}\text { 1st trimester } \\
\text { 2nd \& 3rd trimester } \\
\text { Birth-12 months } \\
13-18 \text { months }\end{array}$ & $\begin{array}{r}0.003 \\
-0.010 \\
-0.003 \\
-0.004 \\
\end{array}$ & $\begin{array}{l}0.013 \\
0.016 \\
0.013 \\
0.011 \\
\end{array}$ & $\begin{array}{l}-0.007 \\
-0.029 \\
-0.017 \\
-0.016 \\
\end{array}$ & $\begin{array}{l}0.015 \\
0.020 \\
0.015 \\
0.013 \\
\end{array}$ & $\begin{array}{l}0.018 \\
0.003 \\
0.009 \\
0.004 \\
\end{array}$ & $\begin{array}{l}0.020 \\
0.026 \\
0.020 \\
0.018 \\
\end{array}$ & $\begin{array}{r}0.001 \\
-0.013 \\
-0.004 \\
-0.011 \\
\end{array}$ & $\begin{array}{l}0.017 \\
0.022 \\
0.017 \\
0.015 \\
\end{array}$ & $\begin{array}{r}0.018 \\
-0.045 \\
-0.013 \\
-0.025 \\
\end{array}$ & $\begin{array}{l}0.044 \\
0.058 \\
0.044 \\
0.039 \\
\end{array}$ \\
\hline THC & $\begin{array}{l}\text { 1st trimester } \\
\text { 2nd \& 3rd trimester } \\
\text { Birth-12 months } \\
\text { 13-18 months }\end{array}$ & $\begin{array}{r}-0.229 \\
0.779 \\
-0.321 \\
-0.343 \\
\end{array}$ & $\begin{array}{l}0.387 \\
0.570 \\
0.548 \\
0.647 \\
\end{array}$ & $\begin{array}{r}0.478 \\
-0.014 \\
0.423 \\
0.094 \\
\end{array}$ & $\begin{array}{l}0.529 \\
0.785 \\
0.750 \\
0.887 \\
\end{array}$ & $\begin{array}{l}0.287 \\
1.745 \\
0.282 \\
0.383 \\
\end{array}$ & $\begin{array}{l}0.700 \\
1.028 \\
0.971 \\
1.172 \\
\end{array}$ & $\begin{array}{l}-0.052 \\
-1.524 \\
-0.391 \\
-1.064 \\
\end{array}$ & $\begin{array}{l}0.574 \\
0.842 \\
0.813 \\
0.958 \\
\end{array}$ & $\begin{array}{r}0.538 \\
1.071 \\
-0.118 \\
-0.809 \\
\end{array}$ & $\begin{array}{l}1.486 \\
2.202 \\
2.107 \\
2.491 \\
\end{array}$ \\
\hline NMHC & $\begin{array}{l}\text { 1st trimester } \\
\text { 2nd \& 3rd trimester } \\
\text { Birth-12 months } \\
\text { 13-18 months }\end{array}$ & $\begin{array}{l}-0.564 \\
-0.585 \\
-0.199 \\
-0.122\end{array}$ & $\begin{array}{l}1.327 \\
1.064 \\
0.897 \\
1.076\end{array}$ & $\begin{array}{l}-0.791 \\
-1.406 \\
-0.307 \\
-0.251\end{array}$ & $\begin{array}{l}1.898 \\
1.518 \\
1.284 \\
1.538\end{array}$ & $\begin{array}{l}2.313 \\
0.830 \\
1.821 \\
2.400\end{array}$ & $\begin{array}{l}2.329 \\
1.875 \\
1.572 \\
1.881\end{array}$ & $\begin{array}{r}0.062 \\
0.024 \\
0.271 \\
-0.211\end{array}$ & $\begin{array}{l}2.116 \\
1.697 \\
1.430 \\
1.717 \\
\end{array}$ & $\begin{array}{r}1.259 \\
-0.956 \\
1.754 \\
2.168\end{array}$ & $\begin{array}{l}5.062 \\
4.061 \\
3.418 \\
4.104\end{array}$ \\
\hline
\end{tabular}

Adjusted for maternal education level, maternal nationality, infant sex, breastfeeding, environmental tobacco smoke exposure, and nursery type. Subject: area * Significant difference $(p<0.05)$. 


\section{Discussion}

Gross motor scores at 6 months of age were negatively associated with the average NMHC levels during the second and third trimesters. In addition, fine motor scores at 18 months of age were negatively associated with the average $\mathrm{SO}_{2}$ exposure during pregnancy and from birth to 12 months of age.

Air pollution can cause cognitive deficits and brain abnormalities, brain damage and neurogeneration [25-27], which has been proven in animal studies [28]. Many studies of hydrocarbons effects did not include methane. Most examined motor vehicle emissions of incompletely combusted hydrocarbons and evaporative fuel losses, which may include known neurotoxic chemicals such as toluene, xylene, ethylene, benzene, $n$-hexane, dimethylpentane, cyclohexane, and stylene. $\mathrm{SO}_{2}$ emissions come from the combustion of fossil fuels, such as diesel fuel, high-sulfur gasoline, and coal. Limited evidence in animal studies alone supports the effect of $\mathrm{SO}_{2}$ on behavioral change. Average $\mathrm{SO}_{2}$ concentrations were relatively low in this study. It is possible that $\mathrm{SO}_{2}$ is a surrogate measure of industrial emissions, and lead is one of the most dangerous neurotoxins found in air pollution. Leaded gasoline has not been used in Taiwan since 2000 and cannot be sold commercially. One study of core blood lead levels in Taiwan found that lead levels were extremely low [29].

There are some specific in our study. First, the air pollution monitoring data were collected by TAQMN and the cohort data were collected from the November and December 2003, and the air pollutant exposure data for each child were linked to the most close to the air-quality monitoring stations and the exposure period from the beginning of the gestational period to eighteen months after birth. The highly relevant of the cohort age and the exposure period could avoid confounder of the season variance were highly accurate for estimating the exposure concentrations at our study subjects' different stages. Second, the birth registration data routinely collected by the government was also highly reliable, providing individual information on mothers and infants that could be important confounding factors.

The subjects' estimated air pollution exposure levels at different stages were based on the average measurements made by the air quality monitoring stations in the subjects' towns of residency. Exposure was assessed at contextual level (i.e., eleven town observations) weighted by timing of the pregnancy resulting could possible minimizes or misclassification the exposure individual variations, the pollutants varying with distance from a traffic road such as $\mathrm{CO}$, hydrocarbons or $\mathrm{NO}_{2}$. But according to the dose-response and the habit of the children care, the result should be underestimating.

Exposure misclassification might come from using outdoor air pollution to represent the exposures for infants who stayed indoors nearly all the time. In one study of indoor and outdoor air pollutants in Taiwan [30] reported that indoor concentrations of air pollutants were highly correlative with outdoor concentrations, while indoor and outdoor climate parameters were less correlated. In addition, one of the most important sources of indoor air pollutants was environmental tobacco smoke (ETS). Previous studies have indicated that prenatal exposure to ETS may affect childhood neurobehavioral development [31]. In our study, we controlled ETS exposure for indoor air pollutants. If any other indoor air pollution existed at the time, it may not have influenced our measurements and could result in an underestimation of the exposure-outcome association.

Most studies that investigated neurobehavioral development in early childhood used the Bayley Scales of Infant Development Mental Development Index [32,33] in this study, we adopted a new 
parental-reported scale to estimate neurobehavioral development in early childhood. The total scores from this scale were used to represent the neurobehavioral development of the studied children. We used the second edition of the Bayley Scale of Infant Development (BSID-II) as the golden standard to test the validity and consistency of the TBCS scale [34,35]. The validity of these scales includes four criteria: a developmental norm, predictive validity, validity and the ability to identify children at relative risk. In our study, the TBCS scale was used to evaluate the neurobehavioral development among these children was relatively advanced or delayed. The self-reported scales may be self-completed differentially by parental wellness may lead to non-differential misclassification. We adjusted the parental education to eliminate the confounder. However, there is still a possible chance to get type I error due to multiple testing. It warrants further research.

This study observes that low-level $\mathrm{SO}_{2}$ exposure prenatally and up to 12 months after birth may impair fine motor development. It is known that $\mathrm{SO}_{2}$ is one of the neurotoxic substances in air pollution. Previous studies about the absorption, distribution, and retention of radioactive $\mathrm{SO}_{2}$ in the mammalian body indicate that radioactive sulfur could be absorbed into the bloodstream of the bronchial tubes and become widely distributed to other body tissues, including the central nervous system [34,35] When $\mathrm{SO}_{2}$ reaches the central nervous system, its biochemical effects change the enzymatic activities of the CNS, including the activities of cofactors (NAD, FAD, and FMN) and nucleic acids (RNA, DNA, and associated proteins) [36] Moreover, $\mathrm{SO}_{2}$ depresses several enzymatic activities of glucose metabolism [37] These enzymes may be associated with neurobehavioral development.

The biological mechanism through which prenatal and postnatal exposure of $\mathrm{SO}_{2}$ could cause adverse childhood neurobehavioral development remains to be explained. However, there are several reports of $\mathrm{SO}_{2}$ 's adverse effects on neurobehavioral development in animal studies. One study indicates that $\mathrm{SO}_{2}$ inhalation by mice and rabbits may increase the incidence of minor skeletal variations among their offspring [38]. In addition, maternal exposure to $\mathrm{SO}_{2}$ could affect neuromuscular coordination and cause functional deficits in the developing offspring. Furthermore, prenatal exposure to $\mathrm{SO}_{2}$ could reduce the levels of several mouse social/agonistic behaviors, such as tail rattling, freezing, and defensive postures. Fine motor performance depends on central nervous system development and neuromuscular coordination. These animal experimental studies provide us with considerable evidence.

In addition, our data about neurobehavioral development at six months of age shows that increased prenatal NMHC exposure during the second and third trimesters in utero were associated with lower gross motor development scores. NMHC is a general term for hydrocarbons other than methane. Many NMHCs are neurotoxic, such as aryl hydrocarbons [39] polycyclic aromatic hydrocarbons [40] and thiophene [41] NMHC's adverse effects on gross motor development at six months of age may be caused by one of the NMHC components or the combined effects of two or more NMHC substances. However, NMHC monitoring data in this study was obtained from only the study areas. To verify the effect of NMHCs on childhood neurobehavioral development, further studies are needed.

There are three principles of early childhood motor development: the cephalocaudal principle, the proximodistal principle, and the hierarchical integration principle. Gross motor skills begin to develop earlier than fine motor skills, and gross skills provide the foundation for fine motor development. We observed that fine motor skills among six-month-old children were minimally variable. This may 
explain why $\mathrm{SO}_{2}$ exposure does not appear to affect fine motor development until eighteen months of age. In addition, gross motor development difference may be more significant at six months of age than at eighteen months. Although we found a no significant adverse effect of NMHC on gross motor skills at eighteen months of age, the adverse effect of NMHC was more significant at six months.

There are some limitations to this study. First, relying on air monitoring data inadequately captures intratown variability. Exposures may vary with, and more than one substance could have an effect over time. Second, development neurotoxicity might be not recognized for years or even decades. Individual vulnerability may vary, and the neurodevelopment scale may not be sufficiently sensitive to detect the effects of low-level exposure. Third, socioeconomic status may be a confounder, as it may relate to the quality and quantity of prenatal and postnatal care a child receives and influence childhood neurobehavioral development $[12,42,43]$. In our analysis, we adjusted for maternal education level, maternal nationality, gestational age, infant sex, breastfeeding, environmental tobacco smoke exposure, and nursery type, as a surrogate measure of the children's home environment and socioeconomic status. Fourth, our study focuses on low levels and long-term exposure effects of air pollutants. This contrasts with previous epidemiological studies, which examined associations between air pollutants and neurobehavioral performance only in experimental acute or short-term exposure [44].

\section{Conclusions}

This study provides evidence that ambient air pollution, even low-level $\mathrm{SO}_{2}$ exposure, during pregnancy and up to 12 months of age is associated with poor subclinical neurodevelopment in early childhood. Further research is necessary to elucidate the causal relationship and provide further evidence of a correlation between the ambient air pollution and neurobehavioral development to improve public health.

\section{Acknowledgments}

This work was supported by the Department of Health, Taiwan (BHP-PHRC-92-4, DOH93-HP-1702, DOH94-HP-1802).

\section{Conflicts of Interest}

The authors declare no conflict of interest.

\section{References}

1. Amitai, Y.; Zlotogorski, Z.; Golan-Katzav, V.; Wexler, A.; Gross, D. Neuropsychological impairment from acute low-level exposure to carbon monoxide. Arch. Neurol. 1998, 55, 845-848.

2. Amitai, N.; Semenova, S.; Markou, A. Cognitive-disruptive effects of the psychotomimetic phencyclidine and attenuation by atypical antipsychotic medications in rats. Psychopharmacology (Berl.) 2007, 193, 521-537.

3. Singh, J. Nitrogen dioxide exposure alters neonatal development. Neurotoxicology 1988, 9, 545-549. 
4. Fiore, M.; Petruzzi, S.; Dell'Omo, G.; Alleva, E. Prenatal sulfur dioxide exposure induces changes in the behavior of adult male mice during agonistic encounters. Neurotoxicol. Teratol. 1998, 20, 543-548.

5. Shih, N.T.; Petering, D.H. Model reactions for the study of the interaction of sulfur dioxide with mammalian organisms. Biochem. Biophys. Res. Commun. 1973, 55, 1319-1325.

6. Tabacova, S.; Nikiforov, B.; Balabaeva, L. Postnatal effects of maternal exposure to nitrogen dioxide. Neurobehav. Toxicol. Teratol. 1985, 7, 785-789.

7. Musi, B.; Dell'Omo, G.; Ricceri, L.; Santucci, D.; Laviola, G.; Bignami, G.; Alleva, E. Effects of acute and continuous ozone $\left(\mathrm{O}_{3}\right)$ exposure on activity/exploration and social behavior of CD-1 mice. Neurotoxicology 1994, 15, 827-835.

8. Rivas-Manzano, P.; Paz, C. Cerebellar morphological alterations in rats induced by prenatal ozone exposure. Neurosci. Lett. 1999, 276, 37-40.

9. Santucci, D.; Sorace, A.; Francia, N.; Aloe, L.; Alleva, E. Prolonged prenatal exposure to low-level ozone affects aggressive behaviour as well as NGF and BDNF levels in the central nervous system of CD-1 mice. Behav. Brain Res. 2006, 166, 124-130.

10. Perera, F.P.; Rauh, V.; Whyatt, R.M.; Tsai, W.Y.; Tang, D.L.; Diaz, D.; Tu, Y.H.; Camann, D.; Kinney, P. Effect of prenatal exposure to airborne polycyclic aromatic hydrocarbons on neurodevelopment in the first 3 years of life among inner-city children. Environ. Health Perspect. 2006, 114, 1287-1292.

11. Perera, F.P.; Li, Z.; Whyatt, R.; Hoepner, L.; Wang, S.; Camann, D.; Rauh, V. Prenatal airborne polycyclic aromatic hydrocarbon exposure and child IQ at age 5 years. Pediatrics 2009, 124, e195-e202.

12. Grandjean, P.; Landrigan, P.J. Developmental neurotoxicity of industrial chemicals. Lancet 2006, 368, 2167-2178.

13. Edwards, S.C.J.W.; Butscher, M.; Camann, D.; Kieltyka, A.; Mroz, E.; Flak, E.; Li, Z.;Wang, S.; Rauh, V.; Perera, F. Prenatal exposure to airborne polycyclic aromatic hyderocarbons and children's intelligence at 5 years of age in a prospective cohort study in poland. Environ. Health Perspect. 2010, 118, 1326-1331.

14. Wang, I.J.; Hsieh, W.S.; Wu, K.Y.; Guo, Y.L.; Hwang, Y.H.; Jee, S.H.; Chen, P.C. Effect of gestational smoke exposure on atopic dermatitis in the offspring. Pediatr. Allergy Immunol. 2008, 19, 580-586.

15. Perera, F.P.W.S.; Vishnevetsky, J.; Zhang, B.; Cole, K.J.; Tang, D.; Rauh, V.; Phillips, D.H. Polycyclic aromatic hydrocarbons-Aromatic DNA adducts in cord blood and behavior scores in New York city children. Environ. Health Perspect. 2011, 119, 1176-1181.

16. Perera, F.P.T.D.; Rauh, V.; Tu, H.Y.; Tsai, W.Y.; Becker, M.; Stein, J.L.; King, J.; del Priore, G.; Lederman, S.A. Relationship between polycyclic aromatic hydrocarbon-DNA adducts, environmental tobacco smoke, and child development in the world trade center cohort. Environ. Health Perspect. 2007, 115, 1497-1452.

17. Tang, D.L.; Li, T.Y.; Liu, J.J., Zhou, Z.J.; Yuan, T.; Chen, Y.H.; Rauh, I.A.; Xie, J.; Perera, F. Effects of prenatal exposure to coal-burning pollutants on children's development in China. Environ. Health Perspect. 2008, 116, 674-679. 
18. Wang, S.; Zhang, J.; Zeng, X.; Zeng, Y.; Chen, S. Association of traffic-related air pollution with children's neurobehavioral functions in Quanzhou, China. Environ. Health Perspect. 2009, 117, 1612-1618.

19. Rodier, P.M. Developing brain as a target of toxicity. Environ. Health Perspect. 1995, 103, $73-76$.

20. Chuang, C.H.; Chang, P.J.; Hsieh, W.S.; Guo, Y.L.; Lin, S.H.; Lin, S.J.; Chen, P.C. The combined effect of employment status and transcultural marriage on breast feeding: A population-based survey in Taiwan. Paediatr. Perinat. Epidemiol. 2007, 21, 319-329.

21. Lung, F.W.; Shu, B.C.; Chiang, T.L.; Lin, S.J. Efficient developmental screening instrument for 6- and 18-month-old children in the Taiwan birth cohort pilot study. Acta Paediatr. 2008, 97 , 1093-1098.

22. Lung, F.W.; Chiang, T.L.; Lin, S.J.; Shu, B.C.; Lee, M.C. Developing and refining the Taiwan birth cohort study (TBCS): Five years of experience. Res. Dev. Disabil. 2011, 32, 2697-2703.

23. Antretter, E.; Dunkel, D.; Osvath, P.; Voros, V.; Fekete, S.; Haring, C. Multilevel modeling was a convenient alternative to common regression designs in longitudinal suicide research. J. Clin. Epidemiol. 2006, 59, 576-586.

24. Williams, B.L.; Pennock-Roman, M.; Suen, H.K.; Magsumbol, M.S.; Ozdenerol, E. Assessing the impact of the local environment on birth outcomes: A case for HLM. J. Expo. Sci. Environ. Epidemiol. 2007, 17, 445-457.

25. Calderon-Garciduenas, L.; Azzarelli, B.; Acuna, H.; Garcia, R.; Gambling, T.M.; Osnaya, N.; Monroy, S.; del Tizapantzi, M.R.; Carson, J.L.; Villarreal-Calderon, A.; et al. Air pollution and brain damage. Toxicol. Pathol. 2002, 30, 373-389.

26. Suglia, S.F.; Gryparis, A.; Schwartz, J.; Wright, R.J. Black carbon associated with cognition among children in a prospective birth cohort study. Epidemiology 2007, 18, S163-S163.

27. Suglia, S.F.; Gryparis, A.; Wright, R.O.; Schwartz, J.; Wright, R.J. Association of black carbon with cognition among children in a prospective birth cohort study. Am. J. Epidemiol. 2008, 167, 280-286.

28. Dorado-Martinez, C.; Paredes-Carbajal, C.; Mascher, D.; Borgonio-Perez, G.; Rivas-Arancibia, S. Effects of different ozone doses on memory, motor activity and lipid peroxidation levels, in rats. Int. J. Neurosci. 2001, 108, 149-161.

29. Hwang, Y.H.; Ko, Y.; Chiang, C.D.; Hsu, S.P.; Lee, Y.H.; Yu, C.H.; Chiou, C.H.; Wang, J.D.; Chuang, H.Y. Transition of cord blood lead level, 1985-2002, in the Taipei area and its determinants after the cease of leaded gasoline use. Environ. Res. 2004, 96, 274-282.

30. Chen, P.C.; Lai, Y.M.; Chan, C.C.; Hwang, J.S.; Yang, C.Y.; Wang, J.D. Short-term effect of ozone on the pulmonary function of children in primary school. Environ. Health Perspect. 1999, 107, 921-925.

31. Slotkin, T.A.; Pinkerton, K.E.; Garofolo, M.C.; Auman, J.T.; McCook, E.C.; Seidler, F.J.. Perinatal exposure to environmental tobacco smoke induces adenylyl cyclase and alters receptor-mediated cell signaling in brain and heart of neonatal rats. Brain Res. 2001, 898, 73-81.

32. Frank, D.A.; Jacobs, R.R.; Beeghly, M.; Augustyn, M.; Bellinger, D.; Cabral, H.; Heeren, T. Level of prenatal cocaine exposure and scores on the bayley scales of infant development: Modifying effects of caregiver, early intervention, and birth weight. Pediatrics 2002, 110, $1143-1152$. 
33. MedoffCooper, B.; Gennaro, S. The correlation of sucking behaviors and bayley scales of infant development at six months of age in vlbw infants. Nurs. Res. 1996, 45, 291-296.

34. Gunnison, A.F.; Benton, A.W. Sulfur dioxide-Sulfite-Interaction with mammalian serum and plasma. Arch. Environ. Health 1971, 22, 381-388.

35. Gunnison, A.F. Sulphite toxicity: A critical review of in vitro and in vivo data. Food Cosmet. Toxicol. 1981, 19, 667-682.

36. Muller, F.; Massey, V. Flavin-sulfite complexes and their structures. J. Biol. Chem. 1969, 244, 4007-4016.

37. Kamogawa, A.; Fukui, T. Inhibition of alpha-glucan phosphorylase by bisulfite competition at phosphate binding-site. Biochim. Biophys. Acta 1973, 302, 158-166.

38. Murray, F.J.; Schwetz, B.A.; Crawford, A.A.; Henck, J.W.; Quast, J.F.; Staples, R.E. Embryotoxicity of inhaled sulfur dioxide and carbon monoxide in mice and rabbits. J. Environ. Sci. Health C 1979, 13, 233-250.

39. Williamson, M.A.; Gasiewicz, T.A.; Opanashuk, L.A. Aryl hydrocarbon receptor expression and activity in cerebellar granule neuroblasts: Implications for development and dioxin neurotoxicity. Toxicol. Sci. 2005, 83, 340-348.

40. Wormley, D.D.; Ramesh, A.; Hood, D.B. Environmental contaminant-mixture effects on cns development, plasticity, and behavior. Toxicol. Appl. Pharmacol. 2004, 197, 49-65.

41. Mori, F.; Tanji, K.; Wakabayashi, K. Thiophene, a sulfur-containing heterocyclic hydrocarbon, causes widespread neuronal degeneration in rats. Neuropathology 2000, 20, 283-288.

42. Mink, P.J.; Goodman, M.; Barraj, L.M.; Imrey, H.; Kelsh, M.A.; Yager, J. Evaluation of uncontrolled confounding in studies of environmental exposures and neurobehavioral testing in children. Epidemiology 2004, 15, 385-393.

43. Ritz, B.; Wilhelm, M.; Zhao, Y. Air pollution and infant death in southern california, 1989-2000. Pediatrics 2006, 118, 493-502.

44. Deschamps, D.; Geraud, C.; Julien, H.; Baud, F.J.; Dally, S. Memory one month after acute carbon monoxide intoxication: A prospective study. Occup. Environ. Med. 2003, 60, 212-216.

(C) 2014 by the authors; licensee MDPI, Basel, Switzerland. This article is an open access article distributed under the terms and conditions of the Creative Commons Attribution license (http://creativecommons.org/licenses/by/3.0/). 